\title{
Metabolic programming of tumor associated macrophages in the context of cancer treatment
}

\author{
Thomas Crezee ${ }^{1}$, Katrin Rabold ${ }^{2,3,4}$, Lisanne de Jong ${ }^{2}$, Martin Jaeger $^{2,4,5}$, Romana T. Netea-Maier ${ }^{5}$ \\ ${ }^{1}$ Department of Pathology, Radboud University Medical Center and Radboud Institute for Molecular Life Sciences, Nijmegen, The Netherlands; \\ ${ }^{2}$ Department of Internal Medicine, Radboud University Medical Center, Nijmegen, The Netherlands; ${ }^{3}$ Radiotherapy and OncoImmunology \\ Laboratory, Department of Radiation Oncology, Radboud University Medical Center, Nijmegen, The Netherlands; ${ }^{4}$ Radboud Institute for Molecular \\ Life Sciences (RIMLS), Radboud University Nijmegen Medical Center, Geert Grooteplein Zuid 28, 6525 GA Nijmegen, The Netherlands; \\ ${ }^{5}$ Department of Internal Medicine, Division of Endocrinology, Radboud University Medical Center, Geert Grooteplein Zuid 8, 6525 GA, Nijmegen, \\ The Netherlands \\ Contributions: (I) Conception and design: RT Netea-Maier; (II) Administrative support: None; (III) Provision of study materials or patients: None; \\ (IV) Collection and assembly of data: None; (V) Data analysis and interpretation: None; (VI) Manuscript writing: All authors; (VII) Final approval of \\ manuscript: All authors. \\ Correspondence to: Romana T. Netea-Maier. Department of Internal Medicine (463), Division of Endocrinology, Radboud University Medical Centre, \\ Geert Grooteplein Zuid 8, 6525 GA Nijmegen, The Netherlands. Email: romana.netea-maier@radboudumc.nl.
}

\begin{abstract}
Tumor associated macrophages (TAMs) are important components of the tumor microenvironment (TME). They are characterized by a remarkable functional plasticity, thereby mostly promoting cancer progression. Changes in immune cell metabolism are paramount for this functional adaptation. Here, we review the functional consequences of the metabolic programming of TAMs and the influence of local and systemic targeted therapies on the metabolic characteristics of the TME that shape the functional phenotype of the TAMs. Understanding these metabolic changes within the context of the cross-talk between the different components of the TME including the TAMs and the tumor cells is an essential step that can pave the way towards identifications of ways to improve responses to different treatments, to overcome resistance to treatments, tumor progression and reduce treatment-specific toxicity.
\end{abstract}

Keywords: Immunotherapy; metabolism; thyroid cancer; tumor associated macrophages; tumor microenvironment (TME)

Submitted Jan 30, 2020. Accepted for publication May 22, 2020.

doi: $10.21037 /$ atm-20-1114

View this article at: http://dx.doi.org/10.21037/atm-20-1114

\section{Introduction}

Inflammation is an emerging hallmark of malignant tumors. The tumor microenvironment (TME) is involved in the pathogenesis and progression of different cancers in general. Characteristically, the TME is a relative nutrient-poor, hypoxic, acidic milieu that can influence the cellular composition and functional phenotype of local as well as infiltrating immune cells. Metabolic shifts within the immune cells result in changes in the immune cells phenotype and function, thereby influencing their effector functions. Additionally, data suggests that the TME also differs between different tumor types, and can change depending on the stage and progression of the disease (1). Because of this, it is hard to predict which changes local cells such as tumor associated macrophages (TAMs) and other immune cells undergo within a specific TME and how those metabolic shifts influence the response to specific treatments. Also, different treatment regiments, including local therapies such as radiotherapy, and systemic agents targeting tumor vascularization and tumor cells such as multikinase inhibitors (MKIs) and mechanistic mammalian target of rapamycin (mTOR) inhibitors can induce (metabolic) changes in the TME (2-4). These changes are 
likely to influence the function of the immune cells in the TME as well. The important question is whether these phenotypic changes synergistically improve the response to therapy or if they are deleterious, leading to more therapy resistance and subsequently tumor progression. Targeting tumor cell and immune cell metabolism in the right way, at the right time, in conjunction with other therapy modalities could be beneficial in patients and be exploited therapeutically. Therefore, understanding the complex interplay between cancer cells and the immune cells on the one hand and the effects of different therapies on this balance on the other hand is paramount in order to identify ways to improve responses to different treatments.

\section{The tumor microenvironment and its metabolic characteristics}

In contrast to normal organs, solid tumors are highly disorganized and heterogenous structures comprising various different cell types. The TME is defined as the surrounding components of a tumor and include parts of the extracellular matrix (ECM), vascularization, such as blood vessels, as well as endothelial cells, fibroblasts and different types of immune cells. The heterogeneous factors and mediators present at the TME promote tumor progression by providing nutrients and signals that lead to immune suppression and thereby generating an anti-inflammatory milieu (5). In addition to containing immune suppressing factors, the TME of most tumors is hypoxic which favors cancer progression and metastasis by upregulating several important genes such as hypoxia-inducible factor 1-alpha (HIF-1 $\alpha)$ (6). During tumor development, the TME is shaped and changed continuously in parallel with the tumor growth. Malignant cells accumulate mutations, leading to clonal diversity and heterogeneity in signal transduction, the epigenome, and gene expression. This induces heterogeneity in differentiation, metabolic and proliferative states across the cancer cell populations (7). Furthermore, tumor cells release various recruitment factors, which result in the infiltration of monocytes and are important mediators in the crosstalk between monocytes and tumor cells (7). Next to these intrinsic changes, other circumstances in the TME, such as proximity to the vasculature, deprivation of and competition for oxygen and nutrients, or the lack of degradation of metabolic waste products, affect the metabolism in both tumor cells and TAMs and the crosstalk between both cell types (Figure 1) (7).

Macrophages are abundant immune cells in the TME of most tumors and their presence is thought to be associated with tumor progression and poor prognosis (8). Because of their specific phenotypical character in cancer, those macrophages were named TAMs. Most TAMs originate from circulating bone marrow and spleen derived monocytes and will differentiate in the TME (9-11). Naturally, macrophages show high levels of functional plasticity which is why numerous phenotypes and intermediate phenotypes have been described at different tissue compartments (12). In general, TAMs immunophenotypically differ from circulating macrophages as they usually show high expression of interleukin-10 (IL10) and low expression of IL-12 as well as higher expression of specific surface receptors such as the mannose receptor (MR, CD206) (13-16). TAMs are often considered M2-like macrophages (or IL-4/IL13-activated) as opposed to the M1-like macrophages (LPS/IFN- $\gamma$-activated). However, since recent insights reveals a spectrum of macrophage activation states rather than a dichotomous M1/M2 model, we will not use the M1/M2 nomenclature in this review but rather describe them according to their source, activators and specific markers if available as currently recommended $(17,18)$. Given the above mentioned characteristics, TAMs strongly contribute to the anti-inflammatory status of the TME. Additionally, TAMs are known to contribute to angiogenesis and metastasis of tumors by upregulating genes responsible for ECM remodelling such as proteases and matrix metallopeptidases ultimately facilitating the escape of tumor cells (19). Interestingly, several animal studies have shown that depletion of macrophages in malignant tumors resulted in decreased tumor progression, thereby leading to a better outcome and prognosis $(20,21)$.

\section{Extracellular circumstances affecting the metabolic characteristics of the TME}

One of the strongest stimuli, inducing the secretion of tumor-derived factors, is hypoxia. During tumor growth, the level of oxygen is strongly reduced due to abnormal tumor vasculature, especially in the center of the neoplasm. Hypoxia triggers HIF-1 $\alpha$ stabilization in neoplastic cells and consequently the release of pro-angiogenic factors, such as growth factors (VEGF, PDGF, PIGF, ANG-2), chemokines (CXCL8, CXCL12), cytokines, (TNFa, IL1 $\beta$, TGF $\beta$ ) and metalloproteases (MMPs), resulting in angiogenesis and thereby cancer cell growth $(22,23)$ as well as monocyte recruitment (24). Furthermore, hypoxia leads to an enhanced glycolytic activity of tumor cells and thereby 


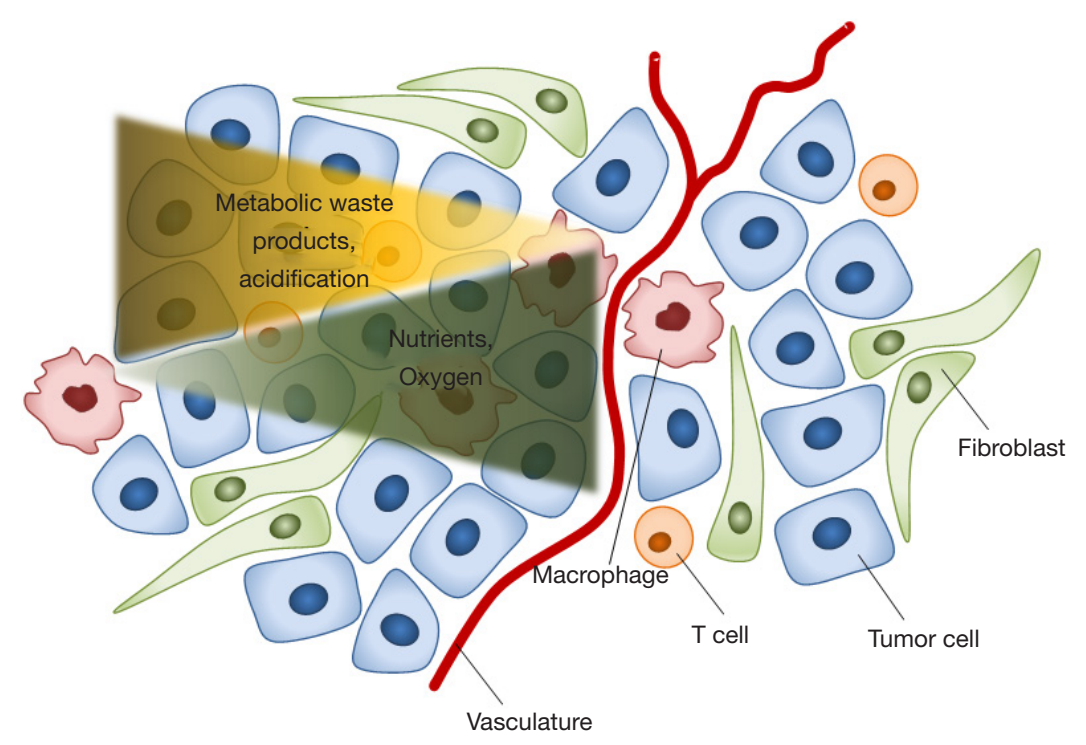

Figure 1 Schematic overview of the tumor microenvironment (TME) containing different cell types that interact with each other. Different gradients regarding the availability of nutrients and oxygen as well as metabolic waste products and the grade of acidification are indicated.

an increased lactate secretion which acidifies the TME and affects the local immune cells (see below) (25).

\section{Tumor cell metabolism}

To sustain the high energy demands of tumor cells required for the high proliferation rate in an oxygen- and nutrientdeprived TME, their metabolism is adapted. It has become evident that metabolic alterations during tumorigenesis encompass all stages of cell-metabolite interaction: (I) increase of metabolite influx, (II) direction of nutrients to metabolic pathways contributing to cellular tumorigenic properties, (III) effect on cellular fate, such as alterations in differentiation by gene-regulation and metabolic interactions with the TME (25).

To fulfill the biosynthetic demands, tumor cells have a high uptake of glucose and glutamine, two principal nutrients that support survival and biosynthesis by maintaining pools of numerous carbon intermediates (26). An elevated consumption of glucose by tumors in comparison to nonproliferating normal cells has been first described by Otto Warburg in 1924 and is now referred to as the "Warburg effect", entailing the shift from oxidative phosphorylation (OXPHOS) as the main source of energy towards anaerobic glycolysis (26). In this, PI3K/Akt signaling acts as a master regulator of glucose uptake by promoting the expression of plasma membrane glucose transporter 1 (GLUT1) (25). Upregulation of GLUT1 and the first enzyme of glycolysis, hexokinase (HK), enables the metabolic shift towards an enhanced glucose uptake and glycolysis (27). Next to glucose, a high demand for glutamine in tumor cells has been first described by Harry Eagle in the 1950s. Eagle showed that the optimal growth of cultured HeLa cells requires substantially more glutamine in culture medium relative to other amino acids (28). In line with this, glutamine depletion from the TME as compared to the corresponding normal tissue is described in numerous tumorigenic contexts (29-32).

Interestingly, proliferating tumor cells convert excess pyruvate as product from glycolysis to lactate rather than transporting it into the mitochondria for OXPHOS. Compared to OXPHOS, anaerobic glycolysis is far less efficient in terms of adenosine triphosphate (ATP) production. However, glycolysis is a robust provider of precursor molecules and reducing equivalents in the form of nicotinamide adenine dinucleotide phosphate (NADPH), whereas tricarboxylic (TCA) cycle activity is the major negative regulator of glucose metabolism. By converting pyruvate to lactate, proliferating tumor cells prevent accumulation of cytosolic NADH and reduce ATP production, promoting glycolysis free from feedback repression by excess mitochondrial ATP generation (25).

Although glycolysis is often described as a single chain of molecular events, leading to the generation of pyruvate, 
several glycolytic intermediates can be shunted into branching pathways as well. The first branch in glycolysis, is the pentose phosphate pathway (PPP), which is often elevated in tumorigenesis and enhances the production of structural components of nucleotides and NADPH. Several other glycolytic intermediates, such as frucotse-6-phosphate and dihydroxyacetone phosphate (DHAP), can be utilized for cellular glycosylation reactions as well as heparan sulfate and hyaluronic acid biosynthesis, or in the biosynthesis of phospholipids, respectively (25), enhancing tumor proliferation.

Moreover, tumor cells show a symbiotic metabolism, as in hypoxic regions of a tumor, cancer cells metabolize glucose through anaerobic glycolysis and express monocarboxylate transporter 4 (MCT4) to release lactate, whilst well-oxygenated cancer cells import the lactate produced by hypoxic cancer cells via MCT1 and consume it to fuel mitochondrial metabolism $(33,34)$.

\section{Metabolism of TAMs}

The metabolic profile of TAMs is very dynamic (Figure 2). In a nutrient-deprived TME, TAMs compete with cancer cells for nutrients such as glucose and therefore undergo changes in their cellular metabolism as well.

Under normoxic conditions, several studies indicate that TAMs have an activated aerobic glycolysis $(35,36)$. Furthermore, metabolic changes in TAMs have also been linked to promotion of tumor invasion and metastasis, linked to an increased glycolytic activity (37).

Next to glucose metabolism, TAMs show an increased glutamine uptake and metabolism (38), whereas glutamine depletion restrains programming towards a pro-tumorigenic phenotype $(39,40)$. Furthermore, some TAMs accumulate intracellular lipids, supporting their immunomodulatory functions (41). Multiple factors involved in lipid metabolism, including enzymes and chaperones are deregulated in TAMs (41-45). Transcriptomic profiling showed high gene expression of lipid metabolism pathways in alveolar resident macrophages, including PPAR- $\gamma$ signaling. Another population defined as TAMs selectively expressed chemokine genes and a population resembling tumor-associated monocytes expressed genes involved in matrix remodeling. These findings underscore the diversity of macrophage phenotypes in cancer (46). Interestingly, PPAR-y signaling is associated with a pro-tumorigenic phenotype of TAMs, as described in an ovarian cancer model (47). Furthermore, TAMs are known to express cyclo-oxygenase 2 (COX2) in vivo and in vitro (48-50). However, the functional relevance of lipid metabolism in TAMs is still not fully understood.

\section{Factors involved in the metabolic and functional programming of TAMs}

Within the TME, TAMs and tumor cells develop a symbiotic relationship which is the result of both tumorderived and TAM-derived factors and the intrinsic adaptation of the cellular metabolism of both cells to the metabolically unfavorable TME.

\section{Chemokines}

Tumor-derived factors are involved in monocyte recruitment, survival, and differentiation within the tumor site. Monocyte chemoattractant protein-1 (MCP1, also known as CCL2) is a tumor- and stromal-derived factor involved in monocyte recruitment (51). Inhibition of the CCL2-CCR2 signaling in a mouse model of breast cancer impaired monocyte infiltration, inhibited metastasis, reduced tumor growth, and depletion of tumor-derived CCL2 inhibited metastatic seeding (52).

Next to CCL2, tumor cells secrete high levels of the growth factor colony stimulating factor-1 (CSF-1), which is involved in recruitment and differentiation of monocytes (53-55). CSF-1 programs monocyte-derived macrophages towards an pro-tumorigenic phenotype coupled to fatty acid oxidation (FAO) upregulation (56) and secretion of pro-tumorigenic and immunosuppressive factors such as epidermal growth factor (EGF) (57) and IL-10 (58).

\section{Hypoxia}

Hypoxia has been shown to induce infiltration of TAMs and reprogramming of macrophages toward the pro-tumorigenic phenotype (59-63), promoting tumor cell proliferation and chemoresistance (64). Under hypoxic conditions, TAMs produce angiogenic factors such as vascular endothelial growth factor (VEGFA). VEGFA stimulates chemotaxis of endothelial cells and macrophages (65). Other angiogenic factors released by TAMs include basic fibroblast growth factor, thymidine phosphorylase, urokinase-type plasminogen activator and adrenomedullin (65-67). Macrophages also promote angiogenesis by physically assisting sprouting blood vessels to augment the complexity of the intra-tumorigenic vascular network (68). 


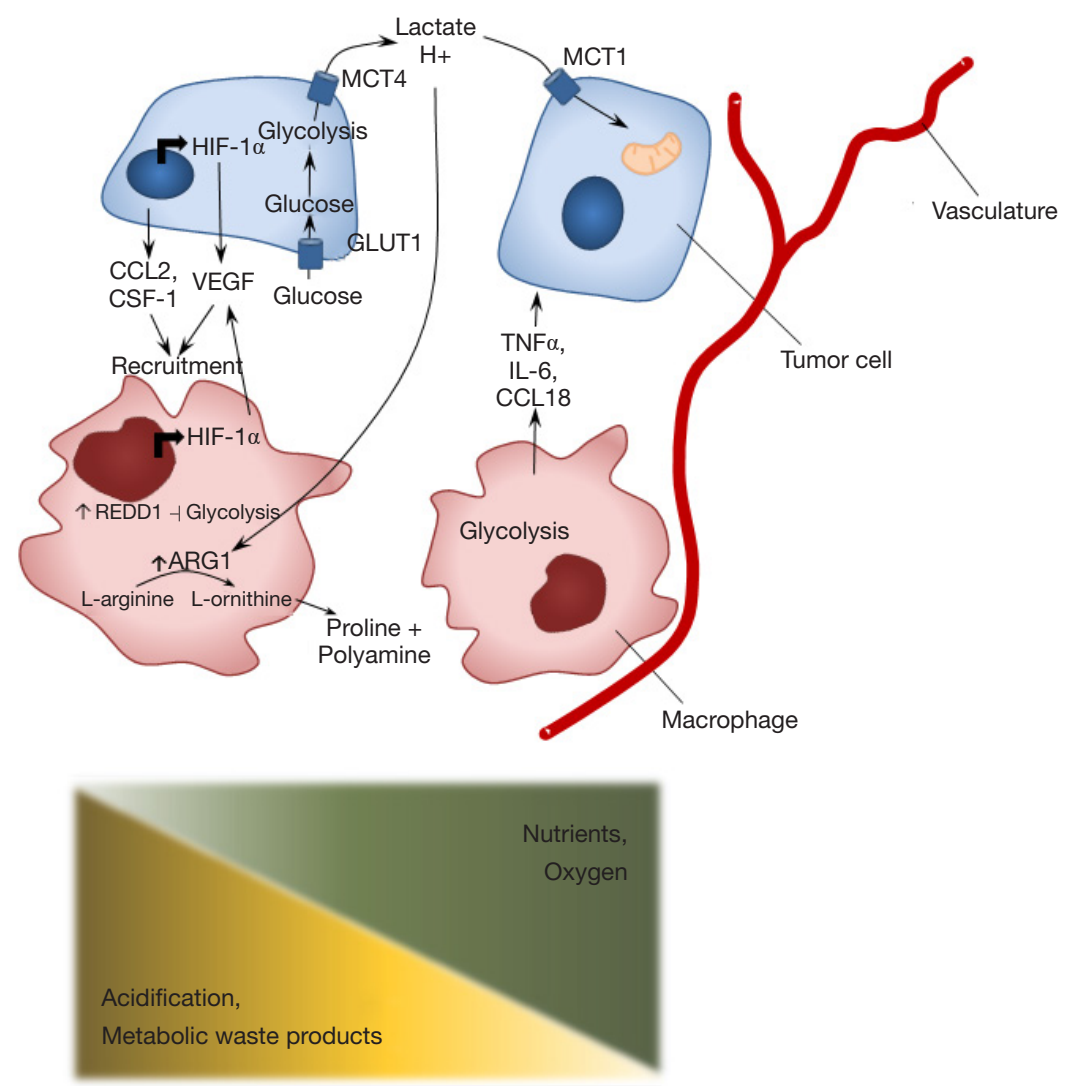

Figure 2 Schematic overview of the metabolic adaptations of tumor cells and (tumor associated) macrophages. Depending on the availability of nutrients and oxygen several metabolic programmes are initiated that allow both cells to adapt to the changing conditions within the TME. Several cytokines and chemokines are involved in these processes and allow both cells to differentiate for adaptation. ARG1, arginase 1; CCL2, monocyte chemoattractant protein-1; CCL18, C-C motif chemokine ligand 18; CSF-1, colony stimulating factor-1; GLUT1, glucose transporter 1; HIF-1 $\alpha$, hypoxia-inducible factor 1-alpha; IL-6, interleukin-6; MCT, monocarboxylate transporter; REDD1, regulated in development and DNA damage responses 1; TNF $\alpha$, tumor necrosis factor alpha; VEGF, vascular endothelial growth factor.

Interestingly, under hypoxic conditions, TAMs upregulate REDD1 (regulated in development and DNA damage responses 1), a negative regulator of mTOR. REDD1mediated mTOR inhibition hinders glycolysis, leaving more glucose for neighboring cells and curtails their excessive angiogenic response, resulting in abnormal blood vessel formation (69).

\section{Lactate}

Extracellular lactate, secreted by tumor cells, functions as signaling molecule which leads the induction of an angiogenic response (70-73). Accumulation of extracellular lactate stimulates the programming of macrophages towards a pro-tumorigenic phenotype and induces expression of VEGF (74-77). Furthermore, the secretion of lactate into the stroma via MCT1 is co-transported with $\mathrm{H}+$, leading to further acidification of the TME. Interestingly, recent in vivo animal studies have shown that differences in function of MCT1 transporter on melanoma cells confer different metastatic potential to these cells. The results suggest that the bidirectional, more efficient handling of lactate by the tumor cells results in a more efficient handling of the oxidative stress and may contribute to the higher metastatic potential in melanoma's (78). Interestingly, acidification of the TME enhances an IL-4 driven phenotype in macrophages and induces a pro-tumor phenotype (79).

\section{Autophagy}

Another process involved in differentiation of macrophages into TAMs is autophagy $(80,81)$. It was found that 
Table 1 Effects of local and systemic therapies on the recruitment, survival and functional reprogramming of TAMs through metabolic mechanisms

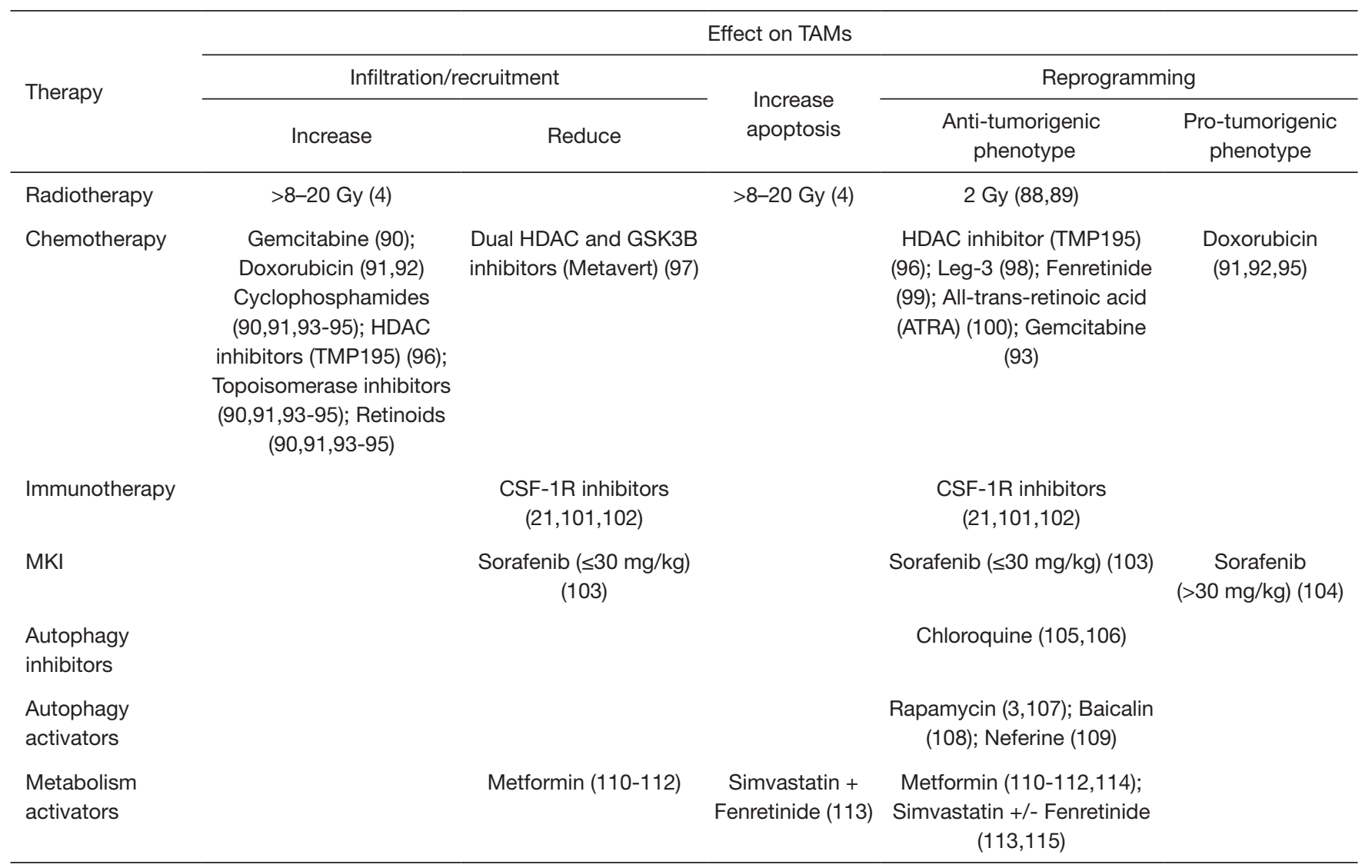

CSF-1R, colony stimulating factor-1 receptor; GSK3B, glycogen synthase kinase 3 beta; Gy, gray; HDAC, histone deacetylase; MKI, multikinase inhibitors; TAMs, tumor-associated macrophages.

autophagy, induced by toll-like receptor 2 (TLR2) signaling, could differentiate bone marrow-derived macrophages into a pro-tumorigenic phenotype in the presence of hepatoma tumor cell condition medium (82). In another study, myeloid-cell specific autophagy was shown to impair antitumorigenic immune responses and promote the survival and accumulation of pro-tumorigenic macrophages in tumor tissues, a process modulated via CSF-1 and transforming growth factor (TGF $\beta$ ) (83). Wen et al. show that tumor cell-released autophagosomes differentiated macrophages into an immunosuppressive phenotype characterized by the expression of programmed cell death protein ligand-1 (PDL1) and IL-10 (84).

Importantly, the effects of metabolic effects of cancer cells on TAMs is not unidirectional. TAMs secrete multiple cytokines with metabolic functions, including IL-6, tumor necrosis factor alpha (TNF $\alpha)$ and CCL18 (85-87). TAM- derived IL-6, TNF $\alpha$ and CCL18 promote tumor cell glycolysis and proliferation (85-87).

\section{Effects of local and systemic therapies on the cross-talk between tumor cells and TAMs and their metabolic reprogramming}

Different local and systemic cancer therapies influence the composition of the TME and the cross-talk between the cellular components of the TME. Some of these effects can be attributed to changes of the metabolic characteristics of the TME through induction of ischemia and hypoxia or through direct effects of these drugs on the cellular metabolism or other intracellular signaling pathways (Table 1). The effect of these therapies on TAMs is not well understood and only scarcely studied, particularly with respect to immunometabolism. 


\section{Radiotherapy}

Upon irradiation, immunosuppressive pathways are activated in the TME and radiation-induced inflammation is caused, subsequently affecting tumor progression. TAMs are recruited and programmed by increased expression of CSF-1 and the destruction of vascularization upon irradiation (4). As immunosuppressive cells, TAMs are involved in the process of radiation-induced inflammation and may promote tumor recurrence (116). HIF-1 $\alpha$ and interferon gamma (IFN- $\gamma$ ) signaling in the TME induces the expression of PD-L1 in TAMs, suppressing antitumor immunity (117). Conversely, regression of tumors at distant sites from the irradiated lesion, called "abscopal" effect, suggest an activation of host immunity (118). Furthermore, radiation-induced tumor cell apoptosis drives differentiation of macrophages into a pro-tumorigenic, anti-inflammatory phenotype $(119,120)$. Interestingly, the effects seem to be dose-dependent, as high doses of irradiation ( $>8 \mathrm{~Gy}$ ) may promote an anti-inflammatory phenotype of macrophages: a dose of 20 Gy induces a phenotype of TAMs with tolerogenic properties (4), whereas low-dose irradiation (2 Gy) skew macrophages towards an anti-tumorigenic phenotype, inducing effective $\mathrm{T}$ cell immunity (88) and influences angiogenic responses in tumor endothelial cells (89).

\section{Chemotherapeutics}

Chemotherapeutic agents are used to directly or indirectly inhibit the proliferation of rapidly growing cells, typically in the context of malignancy. Next to the effects on neoplastic cells, different chemotherapeutic agents among which antimetabolites (e.g., gemcitabine), anti-tumor antibiotics (e.g., anthracyclines), alkylating reagents (e.g., cyclophosphamides), histone deacetylase (HDAC) inhibitors, topoisomerase inhibitors and retinoids have been shown to influence the recruitment and the functional phenotype of TAMs as well. Various chemotherapeutics, including gemcitabine have been reported to induce the recruitment of myeloid cells (90,91,93-95). The immunogenic cell death of neoplastic cells upon chemotherapy leads to modulation of an adaptive immune response (121), which is in line with the discovery that distinct mouse tumors only show an optimal response to anthracyclines in the presence of an intact immune system (122). Interestingly, gemcitabine, even induces a reprograming of TAMs toward an immunostimulatory phenotype in a pancreatic murine tumor model (93).

In contrast, there are discrepant findings in the role of TAMs to the anthracycline Doxorubicin. Whereas some studies show that treatment induces macrophage recruitment, angiogenesis and pro-tumor effects or polarization $(91,92)$, others show that blocking monocyte recruitment by the administration of a CSF-1R-signaling antagonist in a preclinical breast cancer model, enhanced the therapeutic efficacy of chemotherapy $(91,123)$.

Whereas the efficacy of anthracycline-based neoadjuvant chemotherapy in breast cancer patients is associated with the expression levels of the cytokines YKL-39 and CCL18, it is not associated with the amount of TAMs present (124). In line with this, compounds packed in liposomes, such as Long-circulating liposomes (LCL)-encapsulated doxorubicin (Doxil) and LCL-encapsulated prednisolone phosphate (LCL-PLP) have anti-angiogenic and anti-inflammatory effects on TAMs. Interestingly, only LCL-PLP inhibited tumor growth through strong suppressive effects on proangiogenic functions of TAMs (125). Furthermore, a doxorubicin-based prodrug (leg-3) selectively ablated TAMs and resulted in a significant reduction of angiogenic factors and related tumor vessel growth (98).

A selective class IIa HDAC inhibitor, TMP195, induces the recruitment and differentiation of highly phagocytic and stimulatory macrophages. Combining TMP195 with chemotherapy regimens or T-cell checkpoint blockade significantly enhanced the durability of tumor reduction in different studies (96), underscoring a potential role of macrophages in treatment duration. Metavert, a dual inhibitor of glycogen synthase kinase 3 beta (GSK3B) and HDACs, increases killing of drug-resistant pancreatic ductal adenocarcinoma cells by paclitaxel and gemcitabine. Administration of Metavert in vivo leads to beneficial effects such as, decreased TAM infiltration, tumor growth inhibition, prevention of tumor metastasis and a decrease of pro-tumorigenic cytokines (97).

Furthermore, Etoposide, a topoisomerase II-inhibitor, increases cancer cell apoptosis in cells co-cultured with IFN- $\gamma /$ LPS induced macrophages, but reduces apoptosis in the presence of IL-4/IL-13 induced macrophages (126).

Retinoic acid (RA) altered the ability of monocytes to contribute in the tumor angiogenesis process and decreased the ability of TAMs to secrete IL-8 and VEGF (127). Alltrans-retinoic acid (ATRA) abrogated the ability of prostate cancer cell-derived factors to induce a pro-tumorigenic TAM-like phenotype characterized by increased 
proliferation and increased expression of pro-angiogenic, immunosuppressive and pro-metastatic factors. ATRA also inhibited the cancer cell-stimulated proliferation of the pro-tumorigenic macrophages and restored their cytotoxic capacity towards prostate cancer cells (100). Furthermore, fenretinide, a synthetic retinoid, suppresses IL-4/IL13 induced programming of macrophages, shown by the reduced expression of pro-tumorigenic surface markers, the down-regulation of marker genes and the inhibition of protumorigenic macrophage-promoted angiogenesis (99).

Taken together, anthracyclines and retinoids rather have anti-angiogenic effects on TAMs, whereas the effects of HDAC and topoisomerase inhibitors are only scarcely investigated.

\section{Immunotherapies}

In the past decades, substantial evidence is arising that immunotherapy against a broad range of cancers is a suitable alternative to conventional therapies such as chemo- or radiotherapy. In general, this therapeutic approach makes use of existing immunological pathways and cellular interactions to improve the anti-tumorigenic immune response or by blocking undesired interactions of immune cells with cancer cells. To date, several therapies that target specific immune checkpoints have been approved and were proven effective in different forms of cancer. One such checkpoint is the inhibitory interaction between PD-L1 (CD274), expressed by cancer cells, and its receptor PD-1 (CD279) present on different immune cells. T-lymphocyte associated protein-4 (CTLA-4, CD152) is another immune checkpoint efficiently targeted in cancer immunotherapy. The effects of immune checkpoint inhibitors on the TAMs metabolism is largely unknown. Nonetheless, recently, Palaskas et al. showed that PD-1 signaling broadly influences the primary human T-cell metabolism in vitro, shifting it from aerobic glycolysis and glutaminolysis towards utilization of alternative substrates to fuel the TCA cycle. Moreover, the glucose-derived carbon was not derived towards FAO. Furthermore, the study uncovered a block in the nucleoside phosphate de novo synthesis pathway and a reduced mTORC1 signaling (128). Another recent study suggests that immune cell metabolites could be relevant additional markers to predict response to PD-1 blockade therapy (129). Apart from targeting immune checkpoints, inhibiting recruitment of TAMs by blocking the CSF-1/CSF-1R pathway represents a different approach. CSF-1R inhibitors not only have been shown to reduce the number of TAMs, but also reprogramming of TAMs from pro-tumorigenic towards an anti-tumorigenic phenotype $(21,101,102)$.

\section{Multikinase inbibitors (MKI), selective kinase inbibitors and VEGFR targeting drugs}

MKIs have become a promising treatment strategy, since increased intracellular, paracrine and autocrine kinase signaling play a key role in many cancers (2). MKIs often influence tumor vascularization by targeting VEGF receptor (VEGFR) and thereby affect tumor oxygenation (2,130-132). This can also alter the TME to a more immunosuppressive state and inhibit drug uptake in the tumor (2,130-132). Furthermore, VEGFR inhibition can directly affect TAMs, because VEGFR-1 is mainly expressed on anti-inflammatory macrophages and its suppression causes reprogramming towards a more proinflammatory activated phenotype (133). There are several examples of interactions between kinases and VEGFR inhibition and the functional programming of TAMs within the TME, some of which possibly also involving metabolic pathways. The immunomodulatory effects of some MKIs, such as sorafenib, which is broadly used for treatment of hepatocellular carcinoma (HCC), renal cell carcinoma (RCC) and non-medullary thyroid carcinoma (DTC), seem to be dose-dependent (134). As such, treatment of hepatocellular carcinoma (HCC) with sorafenib in low doses $(\leq 30 \mathrm{mg} / \mathrm{kg}$ ), was mostly associated with TAM programming towards a more pro-inflammatory phenotype, possibly through VEGF reduction, miR-101 inhibition or enhanced CCL2 expression (103). Furthermore, sorafenib can inhibit the release of the growth factors mCD163, insulin-like growth factor 1 (IGF-1) and TGF $\beta$ by anti-inflammatory TAMs. Additionally, low dose sorafenib treatment could decrease TAM percentages and increase their expression of four activation markers (FAS-L, chemokine (C-X-C motif) ligand 9 (CXCL-9), CD31, and CD105) (103). However, high doses $(>30 \mathrm{mg} / \mathrm{kg}$ ) impelled immunosuppressive effects in HCC through TAMs by increasing the PDL1 expression (104). Even though, these high doses might not be achievable in humans, TME and immune changes have often been involved in challenging sorafenib resistance (103). Initially, sorafenib dose-dependently inhibits HIF-1 $\alpha$ synthesis (135) leading to downregulated VEGF expression and decreased tumor vascularization. 
However, the hypoxic TME, caused by sustained sorafenib treatment, leads to upregulation of the SDF1- $\alpha /$ CXCR4 axis (136), HIF- $1 \alpha$ and HIF-2 $\alpha$ (137). The increase of HIF- $1 \alpha$ and HIF- $2 \alpha$ has been associated with sorafenib resistance in myeloid leukemia, renal, gastric and hepatoma, because it enhances the expression of target genes involved in mitophagy (BNIP3, NIX), angiogenesis (VEGF), glycolysis (GLUT1, HK2, LDH, PDK1) and proliferation (cyclin D1, c-Myg/PCNA, TGF $\alpha$ /EGFR) (137). Even without hypoxia, sorafenib is known to increase aerobic glycolysis, decrease OXPHOS activity and increase reactive oxygen species (ROS) levels in a dose-dependent manner in hepatoma cells in vitro. This results in higher glucose uptake and lactate production $(138,139)$, thereby altering the TME. It remains to be investigated if sorafenib can also directly shift the metabolism of TAMs in a similar manner.

The immunological effects of vandetanib, the most recently approved MKI for medullary thyroid cancer (MTC), are still mostly unknown. Nonetheless, interesting metabolic effects in highly glycolytic hereditary leiomyomatosis and RCC cells have been reported by Sourbier et al. Vandetanib inhibits the phosphorylation of ABL1, a non-receptor tyrosine kinase, which leads to a reduction of the mTOR-HIF- $1 \alpha$-dependent aerobic glycolysis. In contrast to sorafenib, vandetanib leads to an alteration of the TME by reduced lactate production and glucose uptake, through downregulated Glut1 and Glut4. Additionally, suppressed ABL1 function diminishes the NRF2-dependent antioxidant response which is essential for the survival of ROS-producing tumor cells (140). Future research could clarify whether TAM function is affected by vandetanib, directly or through TME modifications.

\section{Autophagy modulators}

Autophagy is known to have both pro-tumorigenic and antitumorigenic effects in cancer (141). The role of autophagy in cancer might differ in different stages of carcinogenesis, different tumor types and could be related to changes in the TME and treatment. Autophagy activation results in intracellular lysosomal degradation and recycling of proteins and organelles. This process is controlled by autophagy-related genes, regulated by nutrient, energy and stress sensing mechanisms, thereby recycling intracellular components to maintain energy homeostasis and mitochondrial metabolic function inside the cell (141).

\section{Autophagy inhibition}

Chloroquine (CQ), an autophagy inhibitor, was able to reprogram pro-tumorigenic macrophages to an antitumorigenic phenotype. The reprogrammed macrophages presented higher phagocytotic activity towards Hep2 laryngeal tumor cells and re-sensitized Hep-2 cells to cisplatin treatment in vitro (105). Another study by Li et $a l$. showed that CQ abrogated the abnormal angiogenic efficacy of pro-tumorigenic macrophage-conditioned media, thereby decreasing abnormal angiogenesis and lung carcinogenesis (106).

\section{Autophagy stimulation}

Whereas previously mentioned studies demonstrate a beneficial effect of autophagy inhibition on the reprogramming of TAMs, others have shown that the induction of autophagy is the key to reprogramming of TAMs. Shan et al. demonstrate inhibition of programming of mouse RAW 264.7 macrophages to pro-tumorigenic macrophages by autophagy inducer rapamycin. Not only pro-tumorigenic expression was down-regulated, but intracellular ROS generation was also blocked by autophagy induction (3). Another group showed that upregulation of TAM autophagy using rapamycin exhibited effective inhibition of colony formation and proliferation in colorectal cancer cells while inducing apoptosis (107). Like rapamicin, Baicalin, a natural flavonoid, elevated autophagy programmed TAMs towards an anti-tumorigenic phenotype and promoted pro-inflammatory cytokine production. Coculture of HCC cells with baicalin-treated TAMs resulted in reduced proliferation and motility (108).

Neferine, a bisbenzylisoquinoline alkaloid, induced autophagy via inhibition of the mTOR/p70S6K pathway in human umbilical vein endothelial cells and also possessed the ability to suppress pro-tumorigenic macrophage programming (109).

\section{Metabolism modulators}

\section{Metformin}

Metformin, well known for its use in the treatment of diabetes, has been intensively studied for its antitumorigenic properties. Despite the numerous studies, using metformin in clinical trials provided conflicting data $(142,143)$. Metformin-use not only affects the tumor cells, but also influences the TME and TAMs present in the TME. Several studies demonstrate a role of metformin 
in the pro-tumorigenic programming of macrophages. Metformin suppressed IL-13-induced pro-tumorigenic macrophages in vitro, as indicated by a reduced expression of CCL2 and marker CD206 (114). Metformin has also been shown to reduce TAM infiltration, inhibit tumor growth and angiogenesis by skewing TAM programming towards an anti-tumorigenic phenotype (110-112).

\section{Simvastatin}

Simvastatin, a member of the group 3-hydroxy-3methylglutaryl (HMG)-CoA reductase inhibitors, is clinically used for the reduction of hypercholesterolemia. Similar to metformin, simvastatin, has also been studied in relation to cancer. In anaplastic thyroid cancer (ATC), simvastatin has been shown to inhibit proliferation and migration of ATC cells in vitro (144). Additionally, Simvastatin has been shown to have the potential to resensitize drug-resistant cancer cells via reprogramming of TAMs. Simvastatin was able to reprogram TAMs in vitro via cholesterol-associated LXR/ABCA1 regulation, resensitizing non-small cell lung cancer cells to paclitaxel (115). Finally, Simvastatin weakened the TAM-mediated resistance of pancreatic ductal adenocarcinoma cells to gemcitabine (145). A drug combination of simvastatin and fenretinide reprogrammed TAMs in vitro via regulation of the STAT6 pathway as well as induced ROS-mediated mitochondrial apoptosis by inhibiting the Ras/Raf/p-Erk pathway in glioma cells (113).

\section{Future research directions and outstanding questions}

The functional consequences of metabolic programming of TAMs and tumor cells present in the TME has attracted increased attention. Understanding the dynamic metabolic changes and how these affect the functional phenotype of the tumor-infiltrating TAMs is important for the development and improvement of therapies. The same holds true for other immune and non-immune cells which coexist and interplay within the inflammatory TME. This will provide not only a better understanding on the pathophysiology of tumor development and progression, but potentially presents new opportunities for therapy and monitoring of patients with malignant tumors. Next to the conventional therapies, such as radiotherapy, chemotherapy and immunotherapy, novel approaches to target macrophages in the TME could complement the armamentarium of anti-cancer therapy. The latter include strategies to inhibit recruitment and survival of TAMs in the TME, and induce functional reprogramming of these cells towards anti-tumorigenic phenotypes. Such strategies are currently being explored in clinical and translational settings. Despite several limitations related to therapy resistance and loss of sensitivity due to compensatory immunosuppressive mechanisms and toxicity, such approaches show promising results and will most likely be further investigated in clinical trials (146).

Targeting immune cell metabolism to synergize the effect of other systemic or local treatments and overcome therapy resistance is interesting, but at this time it still faces several challenges.

One of the challenges involves the targeting of specific metabolic pathways to the different cell types. Indeed, future work towards the design of delivery systems that could target the metabolic modulators directly towards the desired cell type would be crucial, with the recent progress in nanotechnologies being promising. These developments would automatically help for the second challenge of these therapies: depending on the cell type, what may be favorable for one cell or process, may be deleterious for another, thereby creating either a synergistic or counteracting effect of potential therapeutic metabolic modulators. Finally, as metabolic mediators could affect inadvertently bystander organs and cells, this could lead to side effects, that could be also tacked by targeting the drug to specific cells.

In conclusion, during therapeutic interventions changes in the metabolic landscape of the tumor occur. These changes could result in reprogramming of TAMs into a more anti-tumorigenic phenotype that could be harnessed therapeutically. Future studies are warranted to discover the specific role of cellular metabolic pathways in various cell types in TC, to discover new modulators of these pathways with therapeutic potential, to deliver these molecular modulators to the appropriate cell type, and finally to test and implement these new approaches in patients. In this respect, modulators of immune cell metabolism could be combined with other forms of immunotherapies, such as checkpoint inhibitors, to identify new and more efficient therapeutic strategies. One example of such an approach could be the inhibition of transcription factor c-MAF inducing metabolic programing of TAMs towards a protumoral phenotype, which has been shown to overcome anti-PD-1 therapy resistance in a Lewis lung carcinoma model (147). Only after these studies are performed in the coming years the full potential of metabolic modulators of the immune system will be realized. 


\section{Acknowledgments}

Funding: This work was supported by a grant from the Netherlands Cancer Foundation (KWF) [grant number \#10559, 2017] (Dr. RTNM).

\section{Footnote}

Provenance and Peer Review: This article was commissioned by the Guest Editor (Gaurav Pandey) for the series "Tumor Associated Macrophages in Solid Tumor: Friend or Foe" published in Annals of Translational Medicine. The article was sent for external peer review organized by the Guest Editor and the editorial office.

Conflicts of Interest: All authors have completed the ICMJE uniform disclosure form (available at http:// dx.doi.org/10.21037/atm-20-1114). The series "Tumor Associated Macrophages in Solid Tumor: Friend or Foe" was commissioned by the editorial office without any funding or sponsorship. RTNM reports grants from Netherlands Cancer Foundation (KWF), during the conduct of the study; non-financial support from SOBI, outside the submitted work; In addition, RTNM has a patent Biomarkers for follicular thyroid carcinoma and methods of use issued. The authors have no other conflicts of interest to declare.

Ethical Statement: The authors are accountable for all aspects of the work in ensuring that questions related to the accuracy or integrity of any part of the work are appropriately investigated and resolved.

Open Access Statement: This is an Open Access article distributed in accordance with the Creative Commons Attribution-NonCommercial-NoDerivs 4.0 International License (CC BY-NC-ND 4.0), which permits the noncommercial replication and distribution of the article with the strict proviso that no changes or edits are made and the original work is properly cited (including links to both the formal publication through the relevant DOI and the license). See: https://creativecommons.org/licenses/by-nc-nd/4.0/.

\section{References}

1. Quail DF, Joyce JA. Microenvironmental regulation of tumor progression and metastasis. Nat Med 2013;19:1423-37.

2. Haugen BR, Sherman SI. Evolving approaches to patients with advanced differentiated thyroid cancer. Endocr Rev 2013;34:439-55.

3. Shan M, Qin J, Jin F, et al. Autophagy suppresses isoprenaline-induced M2 macrophage polarization via the ROS/ERK and mTOR signaling pathway. Free Radic Biol Med 2017;110:432-43.

4. Jarosz-Biej M, Smolarczyk R, Cichon T, et al. Tumor Microenvironment as A "Game Changer" in Cancer Radiotherapy. Int J Mol Sci 2019;20:3212.

5. Hanahan D, Coussens LM. Accessories to the crime: functions of cells recruited to the tumor microenvironment. Cancer Cell 2012;21:309-22.

6. Tannahill GM, Curtis AM, Adamik J, et al. Succinate is an inflammatory signal that induces IL-1beta through HIF1alpha. Nature 2013;496:238-42.

7. Lyssiotis CA, Kimmelman AC. Metabolic Interactions in the Tumor Microenvironment. Trends Cell Biol 2017;27:863-75.

8. Zhang QW, Liu L, Gong CY, et al. Prognostic significance of tumor-associated macrophages in solid tumor: a metaanalysis of the literature. PLoS One 2012;7:e50946.

9. Laoui D, Movahedi K, Van Overmeire E, et al. Tumorassociated macrophages in breast cancer: distinct subsets, distinct functions. Int J Dev Biol 2011;55:861-7.

10. Van Overmeire E, Stijlemans B, Heymann F, et al. M-CSF and GM-CSF Receptor Signaling Differentially Regulate Monocyte Maturation and Macrophage Polarization in the Tumor Microenvironment. Cancer Res 2016;76:35-42.

11. Yang L, Zhang Y. Tumor-associated macrophages: from basic research to clinical application. J Hematol Oncol 2017;10:58.

12. Biswas SK, Mantovani A. Macrophage plasticity and interaction with lymphocyte subsets: cancer as a paradigm. Nat Immunol 2010;11:889-96.

13. Mantovani A, Marchesi F, Malesci A, et al. Tumourassociated macrophages as treatment targets in oncology. Nat Rev Clin Oncol 2017;14:399-416.

14. Biswas SK, Allavena P, Mantovani A. Tumor-associated macrophages: functional diversity, clinical significance, and open questions. Semin Immunopathol 2013;35:585-600.

15. Qian BZ, Pollard JW. Macrophage diversity enhances tumor progression and metastasis. Cell 2010;141:39-51.

16. Lewis CE, Pollard JW. Distinct role of macrophages in different tumor microenvironments. Cancer Res 2006;66:605-12.

17. Xue J, Schmidt SV, Sander J, et al. Transcriptome-based network analysis reveals a spectrum model of human macrophage activation. Immunity 2014;40:274-88. 
18. Murray PJ, Allen JE, Biswas SK, et al. Macrophage activation and polarization: nomenclature and experimental guidelines. Immunity 2014;41:14-20.

19. Ojalvo LS, Whittaker CA, Condeelis JS, et al. Gene expression analysis of macrophages that facilitate tumor invasion supports a role for Wnt-signaling in mediating their activity in primary mammary tumors. J Immunol 2010;184:702-12.

20. Zhu Y, Knolhoff BL, Meyer MA, et al. CSF1/CSF1R blockade reprograms tumor-infiltrating macrophages and improves response to T-cell checkpoint immunotherapy in pancreatic cancer models. Cancer Res 2014;74:5057-69.

21. Dammeijer F, Lievense LA, Kaijen-Lambers ME, et al. Depletion of Tumor-Associated Macrophages with a CSF-1R Kinase Inhibitor Enhances Antitumor Immunity and Survival Induced by DC Immunotherapy. Cancer Immunol Res 2017;5:535-46.

22. Bonapace L, Coissieux MM, Wyckoff J, et al. Cessation of CCL2 inhibition accelerates breast cancer metastasis by promoting angiogenesis. Nature 2014;515:130.

23. Bernardini G, Ribatti D, Spinetti G, et al. Analysis of the role of chemokines in angiogenesis. J Immunol Methods 2003;273:83-101.

24. Simons M, Gordon E, Claesson-Welsh L. Mechanisms and regulation of endothelial VEGF receptor signalling. Nat Rev Mol Cell Biol 2016;17:611.

25. Pavlova NN, Thompson CB. The Emerging Hallmarks of Cancer Metabolism. Cell Metab 2016;23:27-47.

26. Warburg O. Über den Stoffwechsel der Carcinomzelle. Naturwissenschaften 1924;12:1131-7.

27. Rathmell JC, Fox CJ, Plas DR, et al. Akt-directed glucose metabolism can prevent Bax conformation change and promote growth factor-independent survival. Mol Cell Biol 2003;23:7315-28.

28. Eagle $H$. The minimum vitamin requirements of the $\mathrm{L}$ and HeLa cells in tissue culture, the production of specific vitamin deficiencies, and their cure. J Exp Med 1955;102:595-600.

29. Márquez J, Sánchez-Jiménez F, Medina MA, et al. Nitrogen metabolism in tumor bearing mice. Arch Biochem Biophys 1989;268:667-75.

30. Rivera S, Azcón-Bieto J, Lopez-Soriano F, et al. Amino acid metabolism in tumour-bearing mice. Biochem $\mathrm{J}$ 1988;249:443-9.

31. Roberts E, Frankel S. Free amino acids in normal and neoplastic tissues of mice as studied by paper chromatography. Cancer Res 1949;9:645-8.

32. Yuneva MO, Fan TW, Allen TD, et al. The metabolic profile of tumors depends on both the responsible genetic lesion and tissue type. Cell Metab 2012;15:157-70.

33. Sonveaux P, Végran F, Schroeder T, et al. Targeting lactate-fueled respiration selectively kills hypoxic tumor cells in mice. J Clin Invest 2008;118:3930-42.

34. Guillaumond F, Leca J, Olivares O, et al. Strengthened glycolysis under hypoxia supports tumor symbiosis and hexosamine biosynthesis in pancreatic adenocarcinoma. Proc Natl Acad Sci U S A 2013;110:3919-24.

35. Arts RJ, Plantinga TS, Tuit S, et al. Transcriptional and metabolic reprogramming induce an inflammatory phenotype in non-medullary thyroid carcinoma-induced macrophages. Oncoimmunology 2016;5:e1229725.

36. Liu D, Chang C, Lu N, et al. Comprehensive Proteomics Analysis Reveals Metabolic Reprogramming of TumorAssociated Macrophages Stimulated by the Tumor Microenvironment. J Proteome Res 2017;16:288-97.

37. Penny HL, Sieow JL, Adriani G, et al. Warburg metabolism in tumor-conditioned macrophages promotes metastasis in human pancreatic ductal adenocarcinoma. Oncoimmunology 2016;5:e1191731.

38. Choi J, Stradmann-Bellinghausen B, Yakubov E, et al. Glioblastoma cells induce differential glutamatergic gene expressions in human tumor-associated microglia/ macrophages and monocyte-derived macrophages. Cancer Biol Ther 2015;16:1205-13.

39. Jha AK, Huang SCC, Sergushichev A, et al. Network integration of parallel metabolic and transcriptional data reveals metabolic modules that regulate macrophage polarization. Immunity 2015;42:419-30.

40. Liu PS, Wang H, Li X, et al. alpha-ketoglutarate orchestrates macrophage activation through metabolic and epigenetic reprogramming. Nat Immunol 2017;18:985-94.

41. Xiang W, Shi R, Kang X, et al. Monoacylglycerol lipase regulates cannabinoid receptor 2-dependent macrophage activation and cancer progression. Nat Commun 2018;9:2574.

42. Miao H, Ou J, Peng Y, et al. Macrophage ABHD5 promotes colorectal cancer growth by suppressing spermidine production by SRM. Nat Commun 2016;7:11716.

43. Niu Z, Shi Q, Zhang W, et al. Caspase-1 cleaves PPARgamma for potentiating the pro-tumor action of TAMs. Nat Commun 2017;8:766.

44. Hao J, Yan F, Zhang Y, et al. Expression of Adipocyte/ Macrophage Fatty Acid-Binding Protein in TumorAssociated Macrophages Promotes Breast Cancer Progression. Cancer Res 2018;78:2343-55. 
45. Rao E, Singh $\mathrm{P}$, Zhai X, et al. Inhibition of tumor growth by a newly-identified activator for epidermal fatty acid binding protein. Oncotarget 2015;6:7815-27.

46. Poczobutt JM, De S, Yadav VK, et al. Expression Profiling of Macrophages Reveals Multiple Populations with Distinct Biological Roles in an Immunocompetent Orthotopic Model of Lung Cancer. J Immunol 2016;196:2847-59.

47. Deng X, Zhang P, Liang T, et al. Ovarian cancer stem cells induce the M2 polarization of macrophages through the PPARgamma and NF-kappaB pathways. Int J Mol Med 2015;36:449-54.

48. Bianchini F, Massi D, Marconi C, et al. Expression of cyclo-oxygenase-2 in macrophages associated with cutaneous melanoma at different stages of progression. Prostaglandins Other Lipid Mediat 2007;83:320-8.

49. Nakao S, Kuwano T, Tsutsumi-Miyahara C, et al. Infiltration of COX-2-expressing macrophages is a prerequisite for IL-1 beta-induced neovascularization and tumor growth. J Clin Invest 2005;115:2979-91.

50. Poczobutt JM, Gijon M, Amin J, et al. Eicosanoid profiling in an orthotopic model of lung cancer progression by mass spectrometry demonstrates selective production of leukotrienes by inflammatory cells of the microenvironment. PLoS One 2013;8:e79633.

51. Lim SY, Yuzhalin AE, Gordon-Weeks AN, et al. Targeting the CCL2-CCR2 signaling axis in cancer metastasis. Oncotarget 2016;7:28697-710.

52. Qian BZ, Li J, Zhang H, et al. CCL2 recruits inflammatory monocytes to facilitate breast-tumour metastasis. Nature 2011;475:222-5.

53. Mizutani K, Sud S, McGregor NA, et al. The chemokine CCL2 increases prostate tumor growth and bone metastasis through macrophage and osteoclast recruitment. Neoplasia 2009;11:1235-42.

54. Scholl SM, Lidereau R, de la Rochefordiere A, et al. Circulating levels of the macrophage colony stimulating factor CSF-1 in primary and metastatic breast cancer patients. A pilot study. Breast Cancer Res Treat 1996;39:275-83.

55. Ao JY, Zhu XD, Chai ZT, et al. Colony-Stimulating Factor 1 Receptor Blockade Inhibits Tumor Growth by Altering the Polarization of Tumor-Associated Macrophages in Hepatocellular Carcinoma. Mol Cancer Ther 2017;16:1544-54.

56. Park J, Lee SE, Hur J, et al. M-CSF from cancer cells induces fatty acid synthase and PPAR / activation in tumor myeloid cells, leading to tumor progression. Cell
Rep 2015;10:1614-25.

57. Wyckoff J, Wang W, Lin EY, et al. A paracrine loop between tumor cells and macrophages is required for tumor cell migration in mammary tumors. Cancer Res 2004;64:7022-9.

58. Ruffell B, Chang-Strachan D, Chan V, et al. Macrophage IL-10 blocks CD8+ T cell-dependent responses to chemotherapy by suppressing IL-12 expression in intratumoral dendritic cells. Cancer Cell 2014;26:623-37.

59. Dubey P, Shrivastava R, Tripathi C, et al. Cyclooxygenase-2 inhibition attenuates hypoxic cancer cells induced m2-polarization of macrophages. Cell Mol Biol (Noisy-le-grand) 2014;60:10-5.

60. McDonald PC, Chafe SC, Dedhar S. Overcoming Hypoxia-Mediated Tumor Progression: Combinatorial Approaches Targeting pH Regulation, Angiogenesis and Immune Dysfunction. Front Cell Dev Biol 2016;4:27.

61. Raggi F, Pelassa S, Pierobon D, et al. Regulation of Human Macrophage M1-M2 Polarization Balance by Hypoxia and the Triggering Receptor Expressed on Myeloid Cells-1. Front Immunol 2017;8:1097.

62. Jiang J, Wang GZ, Wang Y, et al. Hypoxia-induced HMGB1 expression of HCC promotes tumor invasiveness and metastasis via regulating macrophage-derived IL-6. Exp Cell Res 2018;367:81-8.

63. Chen XJ, Wu S, Yan RM, et al. The role of the hypoxiaNrp-1 axis in the activation of M2-like tumor-associated macrophages in the tumor microenvironment of cervical cancer. Mol Carcinog 2019;58:388-97.

64. Song M, Liu T, Shi C, et al. Bioconjugated Manganese Dioxide Nanoparticles Enhance Chemotherapy Response by Priming Tumor-Associated Macrophages toward M1like Phenotype and Attenuating Tumor Hypoxia. ACS Nano 2016;10:633-47.

65. Riabov V, Gudima A, Wang N, et al. Role of tumor associated macrophages in tumor angiogenesis and lymphangiogenesis. Front Physiol 2014;5:75.

66. Chanmee T, Ontong P, Konno K, et al. Tumorassociated macrophages as major players in the tumor microenvironment. Cancers (Basel) 2014;6:1670-90.

67. Zhu C, Kros JM, Cheng C, et al. The contribution of tumor-associated macrophages in glioma neo-angiogenesis and implications for anti-angiogenic strategies. Neuro Oncol 2017;19:1435-46.

68. Squadrito ML, De Palma M. Macrophage regulation of tumor angiogenesis: implications for cancer therapy. Mol Aspects Med 2011;32:123-45.

69. Wenes M, Shang M, Di Matteo M, et al. Macrophage 
metabolism controls tumor blood vessel morphogenesis and metastasis. Cell Metab 2016;24:701-15.

70. Lee DC, Sohn HA, Park ZY, et al. A lactate-induced response to hypoxia. Cell 2015;161:595-609.

71. Ruan GX, Kazlauskas A. Lactate engages receptor tyrosine kinases Axl, Tie2, and vascular endothelial growth factor receptor 2 to activate phosphoinositide 3-kinase/Akt and promote angiogenesis. J Biol Chem 2013;288:21161-72.

72. Végran F, Boidot R, Michiels C, et al. Lactate influx through the endothelial cell monocarboxylate transporter MCT1 supports an NF- B/IL-8 pathway that drives tumor angiogenesis. Cancer Res 2011;71:2550-60.

73. De Saedeleer CJ, Copetti T, Porporato PE, et al. Lactate activates HIF-1 in oxidative but not in Warburgphenotype human tumor cells. PLoS One 2012;7:e46571.

74. Colegio OR, Chu N-Q, Szabo AL, et al. Functional polarization of tumour-associated macrophages by tumourderived lactic acid. Nature 2014;513:559.

75. Hutcheson J, Balaji U, Porembka MR, et al. Immunologic and metabolic features of pancreatic ductal adenocarcinoma define prognostic subtypes of disease. Clin Cancer Res 2016;22:3606-17.

76. Chen P, Zuo H, Xiong H, et al. Gpr132 sensing of lactate mediates tumor-macrophage interplay to promote breast cancer metastasis. Proc Natl Acad Sci U S A 2017;114:580-5.

77. Ohashi T, Akazawa T, Aoki M, et al. Dichloroacetate improves immune dysfunction caused by tumor secreted lactic acid and increases antitumor immunoreactivity. Int J Cancer 2013;133:1107-18.

78. Tasdogan A, Faubert B, Ramesh V, et al. Metabolic heterogeneity confers differences in melanoma metastatic potential. Nature 2020;577:115-20.

79. El-Kenawi A, Gatenbee C, Robertson-Tessi M, et al. Acidity promotes tumour progression by altering macrophage phenotype in prostate cancer. Br J Cancer 2019;121:556-66.

80. Chen P, Cescon M, Bonaldo P. Autophagy-mediated regulation of macrophages and its applications for cancer. Autophagy 2014;10:192-200.

81. Ngabire D, Kim GD. Autophagy and Inflammatory Response in the Tumor Microenvironment. Int J Mol Sci 2017;18.

82. Chang CP, Su YC, Hu CW, et al. TLR2-dependent selective autophagy regulates NF-kappaB lysosomal degradation in hepatoma-derived M2 macrophage differentiation. Cell Death Differ 2013;20:515-23.

83. Jinushi M, Morita T, Xu Z, et al. Autophagy-dependent regulation of tumor metastasis by myeloid cells. PLoS One 2017;12:e0179357.

84. Wen ZF, Liu H, Gao R, et al. Tumor cell-released autophagosomes (TRAPs) promote immunosuppression through induction of M2-like macrophages with increased expression of PD-L1. J Immunother Cancer 2018;6:151.

85. Jeong H, Kim S, Hong B-J, et al. Tumor-associated macrophages enhance tumor hypoxia and aerobic glycolysis. Cancer Res 2019;79:795-806.

86. Zhang Y, Yu G, Chu H, et al. Macrophage-associated PGK1 phosphorylation promotes aerobic glycolysis and tumorigenesis. Mol Cell 2018;71:201-15.e7.

87. Ye H, Zhou Q, Zheng S, et al. Tumor-associated macrophages promote progression and the Warburg effect via CCL18/NF-kB/VCAM-1 pathway in pancreatic ductal adenocarcinoma. Cell Death Dis 2018;9:453.

88. Klug F, Prakash H, Huber PE, et al. Low-dose irradiation programs macrophage differentiation to an iNOS(+)/ M1 phenotype that orchestrates effective T cell immunotherapy. Cancer Cell 2013;24:589-602.

89. Nadella V, Singh S, Jain A, et al. Low dose radiation primed iNOS + M1macrophages modulate angiogenic programming of tumor derived endothelium. Mol Carcinog 2018;57:1664-71.

90. Mitchem JB, Brennan DJ, Knolhoff BL, et al. Targeting tumor-infiltrating macrophages decreases tumorinitiating cells, relieves immunosuppression, and improves chemotherapeutic responses. Cancer Res 2013;73:1128-41.

91. DeNardo DG, Brennan DJ, Rexhepaj E, et al. Leukocyte complexity predicts breast cancer survival and functionally regulates response to chemotherapy. Cancer Discov 2011;1:54-67.

92. Dijkgraaf EM, Heusinkveld M, Tummers B, et al. Chemotherapy alters monocyte differentiation to favor generation of cancer-supporting M2 macrophages in the tumor microenvironment. Cancer Res 2013;73:2480-92.

93. Di Caro G, Cortese N, Castino GF, et al. Dual prognostic significance of tumour-associated macrophages in human pancreatic adenocarcinoma treated or untreated with chemotherapy. Gut 2016;65:1710-20.

94. Nakasone ES, Askautrud HA, Kees T, et al. Imaging tumor-stroma interactions during chemotherapy reveals contributions of the microenvironment to resistance. Cancer Cell 2012;21:488-503.

95. Shree T, Olson OC, Elie BT, et al. Macrophages and cathepsin proteases blunt chemotherapeutic response in breast cancer. Genes Dev 2011;25:2465-79. 
96. Guerriero JL, Sotayo A, Ponichtera HE, et al. Class IIa HDAC inhibition reduces breast tumours and metastases through anti-tumour macrophages. Nature 2017;543:428-32.

97. Edderkaoui M, Chheda C, Soufi B, et al. An Inhibitor of GSK3B and HDACs Kills Pancreatic Cancer Cells and Slows Pancreatic Tumor Growth and Metastasis in Mice. Gastroenterology 2018;155:1985-98.e5.

98. Lin Y, Wei C, Liu Y, et al. Selective ablation of tumorassociated macrophages suppresses metastasis and angiogenesis. Cancer Sci 2013;104:1217-25.

99. Dong R, Gong Y, Meng W, et al. The involvement of M2 macrophage polarization inhibition in fenretinidemediated chemopreventive effects on colon cancer. Cancer Lett 2017;388:43-53.

100. Tsagozis P, Augsten M, Pisa P. All trans-retinoic acid abrogates the pro-tumorigenic phenotype of prostate cancer tumor-associated macrophages. Int Immunopharmacol 2014;23:8-13.

101.Pyonteck SM, Akkari L, Schuhmacher AJ, et al. CSF$1 \mathrm{R}$ inhibition alters macrophage polarization and blocks glioma progression. Nat Med 2013;19:1264-72.

102. Ryder M, Gild M, Hohl TM, et al. Genetic and pharmacological targeting of CSF-1/CSF-1R inhibits tumor-associated macrophages and impairs BRAF-induced thyroid cancer progression. PLoS One 2013;8:e54302.

103.Lin YY, Tan CT, Chen CW, et al. Immunomodulatory effects of current targeted therapies on hepatocellular carcinoma: implication for the future of immunotherapy. Semin Liver Dis 2018;38:379-88.

104. Chen Y, Liu YC, Sung YC, et al. Overcoming sorafenib evasion in hepatocellular carcinoma using CXCR4targeted nanoparticles to co-deliver MEK-inhibitors. Sci Rep 2017;7:44123.

105. Guo Y, Feng Y, Cui X, et al. Autophagy inhibition induces the repolarisation of tumour-associated macrophages and enhances chemosensitivity of laryngeal cancer cells to cisplatin in mice. Cancer Immunol Immunother 2019;68:1909-20.

106. Li GG, Guo ZZ, Ma XF, et al. The M2 macrophages induce autophagic vascular disorder and promote mouse sensitivity to urethane-related lung carcinogenesis. Dev Comp Immunol 2016;59:89-98.

107. Shao LN, Zhu BS, Xing CG, et al. Effects of autophagy regulation of tumor-associated macrophages on radiosensitivity of colorectal cancer cells. Mol Med Rep 2016;13:2661-70.

108. Tan HY, Wang N, Man K, et al. Autophagy-induced RelB/ p52 activation mediates tumour-associated macrophage repolarisation and suppression of hepatocellular carcinoma by natural compound baicalin. Cell Death Dis 2015;6:e1942.

109.Zhang Q, Li Y, Miao C, et al. Anti-angiogenesis effect of Neferine via regulating autophagy and polarization of tumor-associated macrophages in high-grade serous ovarian carcinoma. Cancer Lett 2018;432:144-55.

110.Karnevi E, Andersson R, Rosendahl AH. Tumoureducated macrophages display a mixed polarisation and enhance pancreatic cancer cell invasion. Immunol Cell Biol 2014;92:543-52.

111.Incio J, Suboj P, Chin SM, et al. Metformin Reduces Desmoplasia in Pancreatic Cancer by Reprogramming Stellate Cells and Tumor-Associated Macrophages. PLoS One 2015;10:e0141392.

112. Wang JC, Sun X, Ma Q, et al. Metformin's antitumour and anti-angiogenic activities are mediated by skewing macrophage polarization. J Cell Mol Med 2018;22:3825-36.

113.Mo X, Zheng Z, He Y, et al. Antiglioma via regulating oxidative stress and remodeling tumor-associated macrophage using lactoferrin-mediated biomimetic codelivery of simvastatin/fenretinide. J Control Release 2018;287:12-23.

114. Ding L, Liang G, Yao Z, et al. Metformin prevents cancer metastasis by inhibiting M2-like polarization of tumor associated macrophages. Oncotarget 2015;6:36441-55.

115. Jin H, He Y, Zhao P, et al. Targeting lipid metabolism to overcome EMT-associated drug resistance via integrin beta3/FAK pathway and tumor-associated macrophage repolarization using legumain-activatable delivery. Theranostics 2019;9:265-78.

116.Xu J, Escamilla J, Mok S, et al. CSF1R signaling blockade stanches tumor-infiltrating myeloid cells and improves the efficacy of radiotherapy in prostate cancer. Cancer Res 2013;73:2782-94.

117. Noman MZ, Desantis G, Janji B, et al. PD-L1 is a novel direct target of HIF-1alpha, and its blockade under hypoxia enhanced MDSC-mediated T cell activation. J Exp Med 2014;211:781-90.

118. Durante M, Reppingen N, Held KD. Immunologically augmented cancer treatment using modern radiotherapy. Trends Mol Med 2013;19:565-82.

119. Freire-de-Lima CG, Xiao YQ, Gardai SJ, et al. Apoptotic cells, through transforming growth factor-beta, coordinately induce anti-inflammatory and suppress proinflammatory eicosanoid and $\mathrm{NO}$ synthesis in murine macrophages. J Biol Chem 2006;281:38376-84. 
120. Fadok VA, Bratton DL, Konowal A, et al. Macrophages that have ingested apoptotic cells in vitro inhibit proinflammatory cytokine production through autocrine/ paracrine mechanisms involving TGF-beta, PGE2, and PAF. J Clin Invest 1998;101:890-8.

121. Galluzzi L, Buque A, Kepp O, et al. Immunological Effects of Conventional Chemotherapy and Targeted Anticancer Agents. Cancer Cell 2015;28:690-714.

122. Casares N, Pequignot MO, Tesniere A, et al. Caspasedependent immunogenicity of doxorubicin-induced tumor cell death. J Exp Med 2005;202:1691-701.

123. Williams CB, Yeh ES, Soloff AC. Tumor-associated macrophages: unwitting accomplices in breast cancer malignancy. NPJ Breast Cancer 2016;2:15025.

124.Litviakov N, Tsyganov M, Larionova I, et al. Expression of M2 macrophage markers YKL-39 and CCL18 in breast cancer is associated with the effect of neoadjuvant chemotherapy. Cancer Chemother Pharmacol 2018;82:99-109.

125.Banciu M, Schiffelers RM, Storm G. Investigation into the role of tumor-associated macrophages in the antitumor activity of Doxil. Pharm Res 2008;25:1948-55.

126. Genin M, Clement F, Fattaccioli A, et al. M1 and M2 macrophages derived from THP-1 cells differentially modulate the response of cancer cells to etoposide. BMC Cancer 2015;15:577.

127.Liss C, Fekete MJ, Hasina R, et al. Retinoic acid modulates the ability of macrophages to participate in the induction of the angiogenic phenotype in head and neck squamous cell carcinoma. Int J Cancer 2002;100:283-9.

128. Palaskas NJ, Garcia JD, Shirazi R, et al. Global alteration of T-lymphocyte metabolism by PD-L1 checkpoint involves a block of de novo nucleoside phosphate synthesis. Cell Discov 2019;5:62.

129. Hatae R, Chamoto K, Kim YH, et al. Combination of host immune metabolic biomarkers for the PD-1 blockade cancer immunotherapy. JCI Insight 2020;5:e133501.

130.Huang Y, Yuan J, Righi E, et al. Vascular normalizing doses of antiangiogenic treatment reprogram the immunosuppressive tumor microenvironment and enhance immunotherapy. Proc Natl Acad Sci U S A 2012;109:17561-6.

131. Gao DY, Lin Ts T, Sung YC, et al. CXCR4-targeted lipid-coated PLGA nanoparticles deliver sorafenib and overcome acquired drug resistance in liver cancer. Biomaterials 2015;67:194-203.

132. Kwilas AR, Donahue RN, Tsang KY, et al. Immune consequences of tyrosine kinase inhibitors that synergize with cancer immunotherapy. Cancer Cell Microenviron 2015;2:e677.

133. Lacal PM, Graziani G. Therapeutic implication of vascular endothelial growth factor receptor-1 (VEGFR-1) targeting in cancer cells and tumor microenvironment by competitive and non-competitive inhibitors. Pharmacol Res 2018;136:97-107.

134. Iyer RV, Maguire O, Kim M, et al. Dose-Dependent Sorafenib-Induced Immunosuppression Is Associated with Aberrant NFAT Activation and Expression of PD-1 in T Cells. Cancers (Basel) 2019;11:681.

135. Liu LP, Ho RL, Chen GG, et al. Sorafenib inhibits hypoxia-inducible factor- $1 \alpha$ synthesis: implications for antiangiogenic activity in hepatocellular carcinoma. Clin Cancer Res 2012;18:5662-71.

136. Ramjiawan RR, Griffioen AW, Duda DG. Antiangiogenesis for cancer revisited: Is there a role for combinations with immunotherapy? Angiogenesis 2017;20:185-204.

137. Méndez-Blanco C, Fondevila F, García-Palomo A, et al. Sorafenib resistance in hepatocarcinoma: role of hypoxiainducible factors. Exp Mol Med 2018;50:1-9.

138. Tesori V, Piscaglia AC, Samengo D, et al. The multikinase inhibitor Sorafenib enhances glycolysis and synergizes with glycolysis blockade for cancer cell killing. Sci Rep 2015;5:9149.

139.Feng J, Wu L, Ji J, et al. PKM2 is the target of proanthocyanidin B2 during the inhibition of hepatocellular carcinoma. J Exp Clin Cancer Res 2019;38:204.

140.Sourbier C, Ricketts CJ, Matsumoto S, et al. Targeting ABL1-mediated oxidative stress adaptation in fumarate hydratase-deficient cancer. Cancer Cell 2014;26:840-50.

141. White E, Mehnert JM, Chan CS. Autophagy, Metabolism, and Cancer. Clin Cancer Res 2015;21:5037-46.

142. Gong J, Kelekar G, Shen J, et al. The expanding role of metformin in cancer: an update on antitumor mechanisms and clinical development. Target Oncol 2016;11:447-67.

143. Kurelac I, Umesh Ganesh N, Iorio M, et al. The multifaceted effects of metformin on tumor microenvironment. Semin Cell Dev Biol 2020;98:90-7.

144. Chen MC, Tsai YC, Tseng JH, et al. Simvastatin Inhibits Cell Proliferation and Migration in Human Anaplastic Thyroid Cancer. Int J Mol Sci 2017;18:2690.

145.Xian G, Zhao J, Qin C, et al. Simvastatin attenuates macrophage-mediated gemcitabine resistance of pancreatic ductal adenocarcinoma by regulating the TGF-beta1/Gfi1 axis. Cancer Lett 2017;385:65-74. 
146. Cassetta L, Pollard JW. Targeting macrophages: therapeutic approaches in cancer. Nat Rev Drug Discov 2018;17:887-904.

Cite this article as: Crezee T, Rabold K, de Jong L, Jaeger M, Netea-Maier RT. Metabolic programming of tumor associated macrophages in the context of cancer treatment. Ann Transl Med 2020;8(16):1028. doi: 10.21037/atm-20-1114
147.Liu M, Tong Z, Ding C, et al. Transcription factor c-Maf is a checkpoint that programs macrophages in lung cancer. J Clin Invest 2020;130:2081-96. 